\title{
Ulcerative Colitis associated with Sclerosing Cholangitis and Autoimmune Hepatitis
}

\author{
Hoduț A, Simedrea I, Sabău I, Belei Oana, Babi lonela \\ First Pediatric Clinic, Faculty of Medicine, "Victor Babeș" University of Medicine and Pharmacy, Timișoara, Romania
}

\begin{abstract}
Introduction: Ulcerative colitis is a chronic intestinal inflammation, part of inflammatory bowel disease, which also includes Crohn's disease. Both have extraintestinal manifestations, but those that tend to occur more commonly with ulcerative colitis include chronic active hepatitis, pyoderma gangrenosum and ankylosing spondylitis. Many individuals present with overlapping non-diagnostic features of more than one of these conditions that is referred to in the literature as autoimmune overlap syndrome. Sclerosing cholangitis associated with IBD is often referred to as overlap syndrome.
\end{abstract}

Material and methods: We present the case of a 15-year-old female, with an association between ulcerative colitis, primitive sclerosing cholalangitis and autoimmune hepatitis. She was admitted for: diarrheic bloody stools, abdominal pain, diminished appetite, headache and aphthous stomatitis. Blood sample analysis revealed: hypochromic anemia, iron deficiency, high levels of transaminase, abnormal protein electrophoresis, positive anti-neutrophil cytoplasmic antibodies and anti-smooth muscle antibodies, high level of faecal calprotectin, modified biliary tract on imaging of digestive system and suggestive modifications of colic mucosa for ulcerative colitis. We administered treatment with Arginine Chloride 5\%, Sorbitol 10\%, Aspartic acid, Vitamin B6, Ursodeoxycholic acid, 5-aminosalicylic acid.

Results: With the administered therapy the evolution was good, macroscopic blood disappeared from stools, and tests for blood trace in stool were also negative.

Conclusions: The patient had simultaneous onset of diarrhea with bloody stools and extraintestinal manifestations. Immunological markers didn't fully match any of the associated diseases, so we concluded that there was an overlap syndrome. Budesonide was effective on both hepatic and intestinal disease.

Keywords: ulcerative colitis, overlap syndrome, calprotectin, budesonide, 5-aminosalicylic acid, ursodeoxycholic acid

Received: 13 April 2012

\section{Introduction}

Ulcerative colitis (UC) is a chronic intestinal inflammation, characterized by unpredictable exacerbations and remissions, it is localized to the colon and spares the upper gastrointestinal tract. It is part of inflammatory bowel disease (IBD), which also includes Crohn's disease (CD). Both have extraintestinal manifestations, but some of these tend to occur more commonly with UC than with CD. This group of manifestations includes chronic active hepatitis, pyoderma gangrenosum and ankylosing spondylitis [1].

Sclerosing cholalangitis is a common hepatobiliary disease associated with IBD, which is characterized by progressive inflammation and fibrosis of intra- and extrahepatic bile ducts. Sclerosing cholalangitis associated with IBD is often referred as overlap syndrome [2].

There is currently no agreement on what constitutes an autoimmune overlap syndrome [3]. Two distinct types of overlap syndrome can be considered: the Crossover and the True Overlap [4]. Autoimmune hepatitis (AIH), formerly known as chronic active hepatitis $(\mathrm{CAH})$ is a progressive inflammatory liver disease mainly affecting young women [5]. Some patients present with features of an autoimmune liver condition that do not conform uniquely to any of the established criteria for the diagnosis of AIH, primary

Correspondence to: Andrei Hodut

E-mail: andreihodut@yahoo.com biliary cirrhosis (PBC), or primary sclerosing cholangitis (PSC) [3].

\section{Material and method}

We present the case of a 15-year-old female, from urban habit, with an association between ulcerative colitis, primitive sclerosing cholalangitis and autoimmune hepatitis - described in the literature as autoimmune overlap syndrome. We have the written agreement, signed by her mother, to publish this article.

The patient came to First Pediatric Clinic Timișoara, being admitted in November 2010, at the age of 15 years and 5 months, for diarrheic bloody stools (4-5 diarrheic stools/ day, 2-3 of them with fresh blood) abdominal pain (epigastric and hypogastric), diminished appetite, headache and aphthous stomatitis.

The patient was diagnosed 4 years earlier with irritable bowel syndrome (IBS) by the general practioner when she presented from time to time abdominal pain and diarrheic stools.

Physical examination at first admission to our clinic revealed a moderately influenced condition and growth failure. Data were pertained to 2007 WHO growth standards. (height $=157 \mathrm{~cm}-$ percentile $15-25 \%$, weight $=42 \mathrm{~kg}, \mathrm{BMI}=17.03 \mathrm{~kg} / \mathrm{m}^{2}-$ percentile $\left.5-15 \%\right)$. The oral mucosa presented aphthous lesions, the skin was pale. The abdomen was tender at palpation, the cardio- 
respiratory system was normal, sexual development was normal too: menarche at the age of 12 , normal menstrual cycles ever since. During the first period of hospitalization she had semi-liquid stools (3-4/day), of these 1-2 with fresh blood.

Blood sample analysis revealed hypochromic anemia with iron deficiency - Hemoglobin [HGB] $10.2 \mathrm{~g} / \mathrm{dl}$, hematocrit [HCT] 34.7\%, mean corpuscular volume [MCV] $84.8 \mathrm{fL}$, mean corpuscular hemoglobin $[\mathrm{MCH}] 24.9 \mathrm{pg}$, mean corpuscular hemoglobin concentration [MCHC] $29.4 \mathrm{~g} / \mathrm{dl}$, blood iron concentration $[\mathrm{Fe}] 4.8 \mu \mathrm{mol} / \mathrm{l}$; normal leucocytes [WBC] number $\left(5,200 / \mathrm{mm}^{3}\right)$, with neutropenia (47.0\%) and monocytosis (11.5\%), high inflammation (erythrocytes sedimentation rates [ESR] $80 \mathrm{~mm} / \mathrm{h}$, but normal C-reactive protein [CRP] $0.63 \mathrm{mg} / \mathrm{l}$ and fibrinogen $[\mathrm{Fbg}] 3.24 \mathrm{~g} / \mathrm{l})$. Hepatic blood tests revealed high levels of transaminase (alanine aminotransferase [ALAT] 195 IU/l, aspartate aminotransferase [ASAT] $150 \mathrm{IU} / \mathrm{l}$, gammaglutamyl transpeptidase [GGT] 139 IU/l. Alkaline phosphatase [ALP] levels was normal (75 IU/l), both direct and indirect bilirubinemia were in normal range. Protein level was a bit higher than normal at $84.6 \mathrm{~g} / \mathrm{l}$, with an abnormal electrophoresis: decreased Albumin [Alb] 46.1\% and Albu$\mathrm{min} /$ Globulin ratio $[\mathrm{A} / \mathrm{G}]$ 0.85; Immunoglobulin quantitation was also abnormal - increased Immunoglobulin G [IgG] $28.95 \mathrm{~g} / \mathrm{l}$, and normal ranges of Immunoglobulin A [IgA] $2.33 \mathrm{~g} / \mathrm{l}$ and Immunoglobulin M [IgM] $1.01 \mathrm{~g} / \mathrm{l}$. Immunological markers such as: rheumatoid factor, lupic cell, anti-nuclear antibodies (ANA), anti-streptolysin O antibody (ASLO), anti-liver/kidney microsomal antibodies (LKM), antimitochondrial antibodies (AMA), antibodies to Saccharomyces cerevisiae (ASCA) were all negative. Instead, we found positive levels for anti-neutrophil cytoplasmic antibodies (ANCA) 1/80 and for anti-smooth muscle antibodies (SMA) 1/160. Faecal calprotectin level was high $(300 \mu \mathrm{g} / \mathrm{g})$. We used two types of tests: one was a quantitative assay determined by one laboratory, that expressed results in $\mu \mathrm{g} / \mathrm{g}$, the other one was qualitative, a quick test. Both test had four levels for interpretation, from no inflammation to very high inflammation of the gastrointestinal tube. Alpha 1-antitrypsin level was not modified $1.4 \mathrm{~g} / \mathrm{l}$.

Bacteriological tests (cultures from the nose, pharynx, bloodstream, urine, and stool - even tests for enteric pathogens), ova, parasites and virusological tests (hepatic viruses $\mathrm{A}, \mathrm{B}, \mathrm{C}$, cytomegalovirus and Human Immunodeficiency Virus) were all negative.

Thyroid hormones were normal. Ultrasound showed an inhomogeneous structure of left thyroidal lobe.

Imaging of the digestive system revealed modified biliary tract: ultrasonography observed an enlarged gallbladder and bile duct ( $6 \mathrm{~mm}$ in diameter), with hyperlucent walls, a small infundibular septum. Intrahepatic biliary ducts presented the shotgun sign. All other abdominal organs appeared normal. Magnetic resonance imaging (MRI) of the abdomen was normal. MRI with three dimensional reconstructions [MRI-3D] showed a main biliary duct

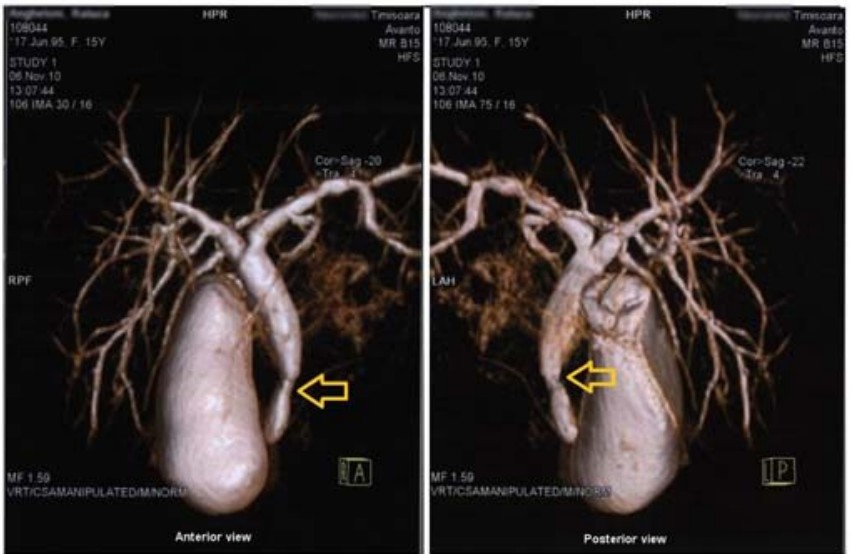

Fig. 1. MRI with 3D reconstruction of biliary ducts $(\varnothing \sim 10 \mathrm{~mm})$. Arrow: narrowing of the main biliary duct $(L \sim 1 \mathrm{~cm}, \varnothing \sim 3 \mathrm{~mm})$ in the distal half

with a diameter of approximately $10 \mathrm{~mm}$. The distal half presented a narrowing with a diameter of $3 \mathrm{~mm}$ and length of $10 \mathrm{~mm}$ Proximal from this the biliary ducts were slightly enlarged and with some irregularities inside the walls. Wirsung's duct was normal (Figure 1).

Endoscopic examination was performed up into the distal $10 \mathrm{~cm}$ ileum. Marked erythema was observed wyh absent vascular pattern, high friability, erosions in most of the segments except for the anal canal and cecum that appared normal. Splenic flexure and the ascending colon had, beside the already described lesions, some small red hemorrhagic spots that were not spontaneously bleeding at the examination moment. Pathology exam of bioptic fragments taken from colon and ileum observed piohemorrhagic ileitis with thrombosis, important fibrousmixo-collagenic repair especially in the submucosal layer. Fragments taken from colon revealed modification of nonspecific chronic pio-hemorrhagic colitis.Parents requested postponement of liver biopsy and so we had to support diagnosis without it.

We established the diagnoses of ulcerative pancolitis, sclerosing cholangitis and autoimmune hepatitis.

Pediatric Ulcerative Colitis Activity Index (PUCAI)[6] at the admission time counted 45 points: 5 for each of the following: abdominal pain - [that can be ignored], number of stools per day - [3-5/day] and activity level - [occasional limitation of activity] and 10 for rectal bleeding - [small amount in less than $50 \%$ of stools], stool consistency - [completely unformed], nocturnal stools - [any episode causing weakening]. Mayo score was of 7 points: 2 for each of stool frequency, endoscopic findings and physician's global assessment, 1 for rectal bleeding [7].

We intended to induce remission. For this we first controlled the hepatic problems - we recommended a diet for hepatic and intestinal protection, we administered Arginine Chloride 5\% + Sorbitol 10\% $250 \mathrm{ml} /$ day in endovenous infusion, Aspartic acid $500 \mathrm{mg} /$ day + Vitamin B6 $250 \mathrm{mg} /$ day also in endovenous infusion, Ursodeoxycholic acid (UDCA) $750 \mathrm{mg} /$ day; for gastric symptoms we gave 


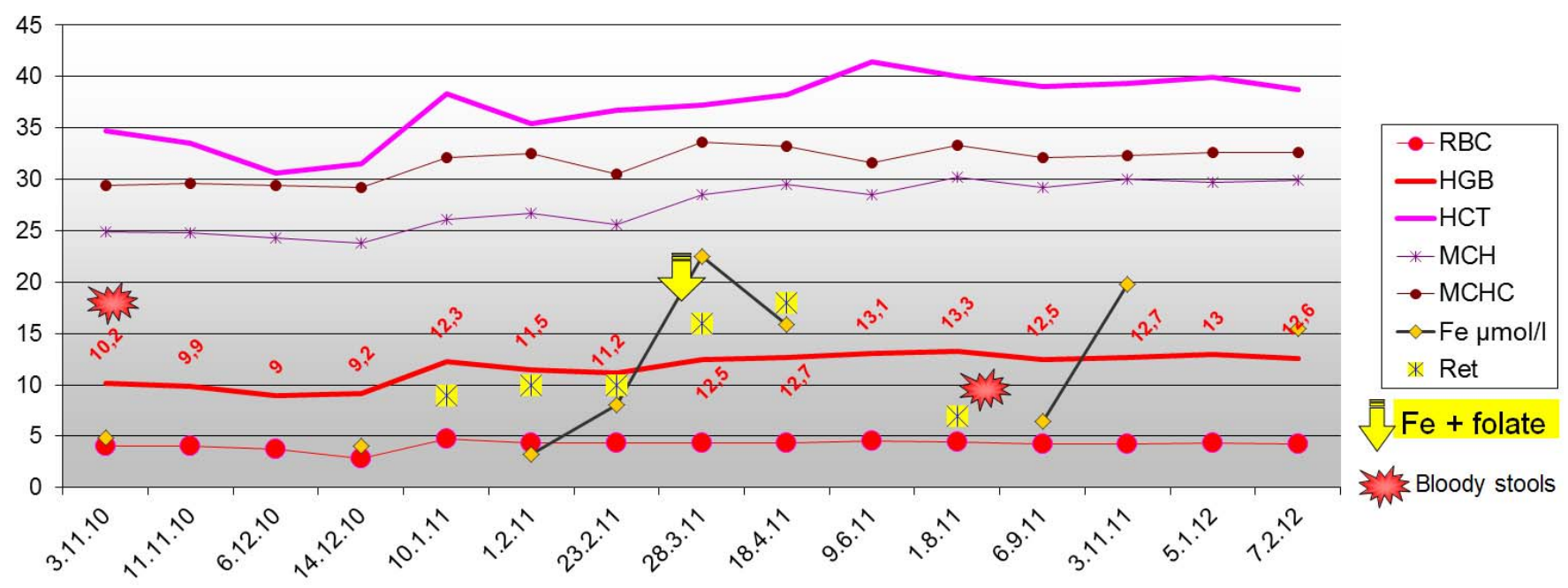

Fig. 2. Blood tests for anemia

Esomeprazole $40 \mathrm{mg} /$ day; we also administered Trimebutine maleate $100 \mathrm{mg} /$ day and probiotics all of them in oral administration.

With this therapy transaminase decreased and than normalized in two weeks.

At the beginning of December 2010 we initiated specific therapy for ulcerative colitis with Budesonide capsule $9 \mathrm{mg} /$ day and continued treatment with UDCA, Esomeprazole and probiotics. We added to treatment zinc $15 \mathrm{mg} /$ day, iron glutamate $100 \mathrm{mg} /$ day, folic acid (B9) $5 \mathrm{mg} /$ day.

In March 2011 we added to treatment 5-aminosalicylic acid (5-ASA) and continued to decrease budesonide so that in February 2012 she receives only $3 \mathrm{mg} /$ day, combined with 5-ASA 3 g/day.

\section{Results}

With this therapy the evolution was good, symptoms regressed and than disappeared. Macroscopic blood disappeared from stools, and tests for blood trace in stool were also negative. In September 2011 fresh blood reappeared in stools.

Blood iron normalized by end of February and hemoglobin level was corrected by the end of March 2011 (HGB $=12.5 \mathrm{~g} / \mathrm{dl}$ ). Blood iron decreased when blood reappeared in stools (Figure 2).

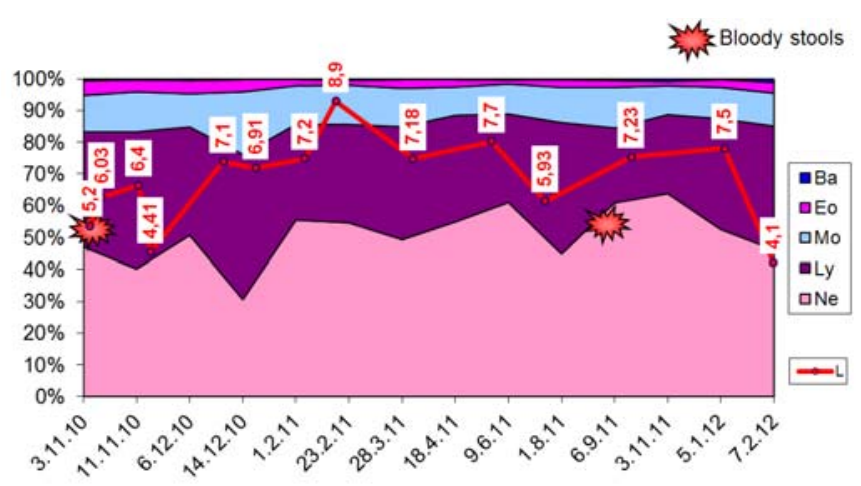

Fig. 3. Leukocyte formula (\%)
Leucocytes number was normal except for December 2010 and February 2012 when there was a slight leucopenia. Leucocytes formula instead showed constant monocytosis with neutropenia or lymphopenia. In February 2012 there was only a discrete basophilia (Figure 3).

Inflammation tests ESR and fecal calprotectin decreased slowly till we introduced 5-aminosalicylic acid (5-ASA) at beginning of March 2011. After this moment ESR decreased more rapidly, became negative in June, than rose again in September 2011. Fecal calprotectin instead became quickly negative by the end of March and attained a high level again $(>214 \mu \mathrm{g} / \mathrm{g})$ only two weeks later. After that regressed slowly, and normalized in January 2012. It was only slightly elevated when blood reappeared in stools. CRP was all this time negative (Figures 4, 5). Hepatic tests normalized quickly in November 2010. In September 2011 ASAT and ALAT slightly increased but GGT decreased under the normal value. One month later all the three were back in normal ranges. ALP levels and bilirubinemia were normal (Figure 6). Gamma globulin levels were high all the time and returned to normal in February 2012. We repeated ANCA and SMA at the beginning of February 2011, both were negative. One year later we repeated rheumatoid factor and anti-nuclear antibodies (ANA) which were also negative.

Repeated ultrasonography of digestive system showed the same modifications of biliary ducts. In early February 2011 macroscopic aspect of mucosa was improved, but were present all along the colon. Pathology exam observed chronic ulcerative colitis lesions with inflammation, lymphoid reaction, ulceration, fibrosis. In fragments from ileum, microscopy was suggestive for backwash ileitis. Mayo endoscopic score decreased, and so did PUCAI score, to rise again in September 2011. After this it decreased at a low level (Figure 7).

\section{Discussions}

IBD have poorly understood etiologies, an abnormal im- 


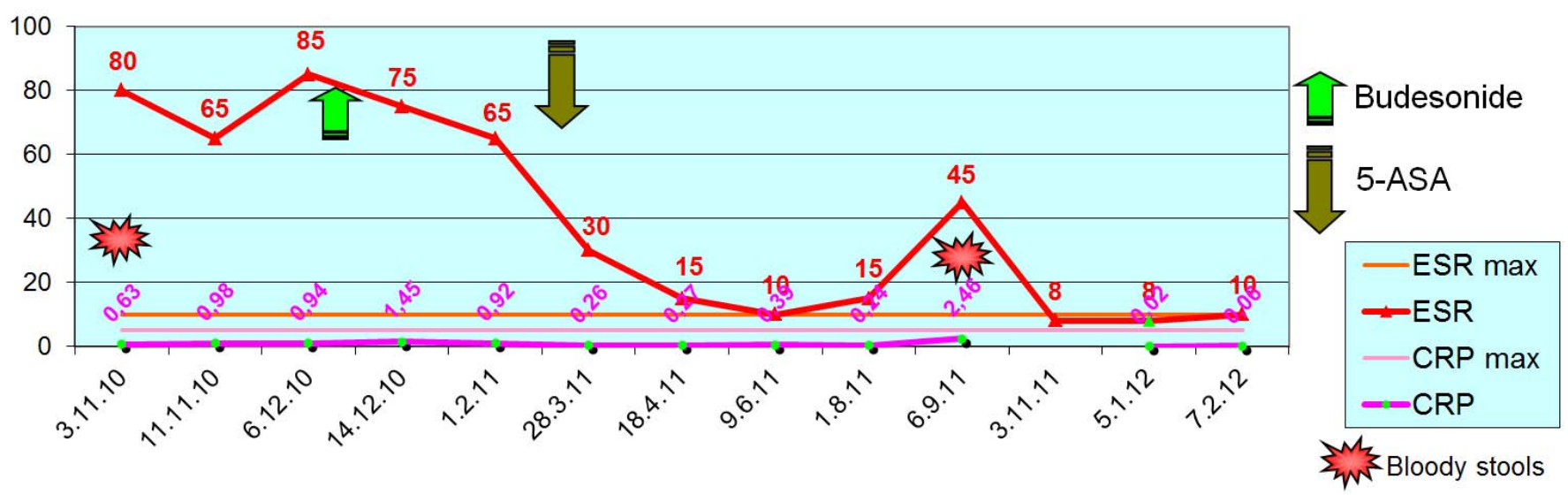

Fig. 4. Inflammatory tests (ESR, CRP)

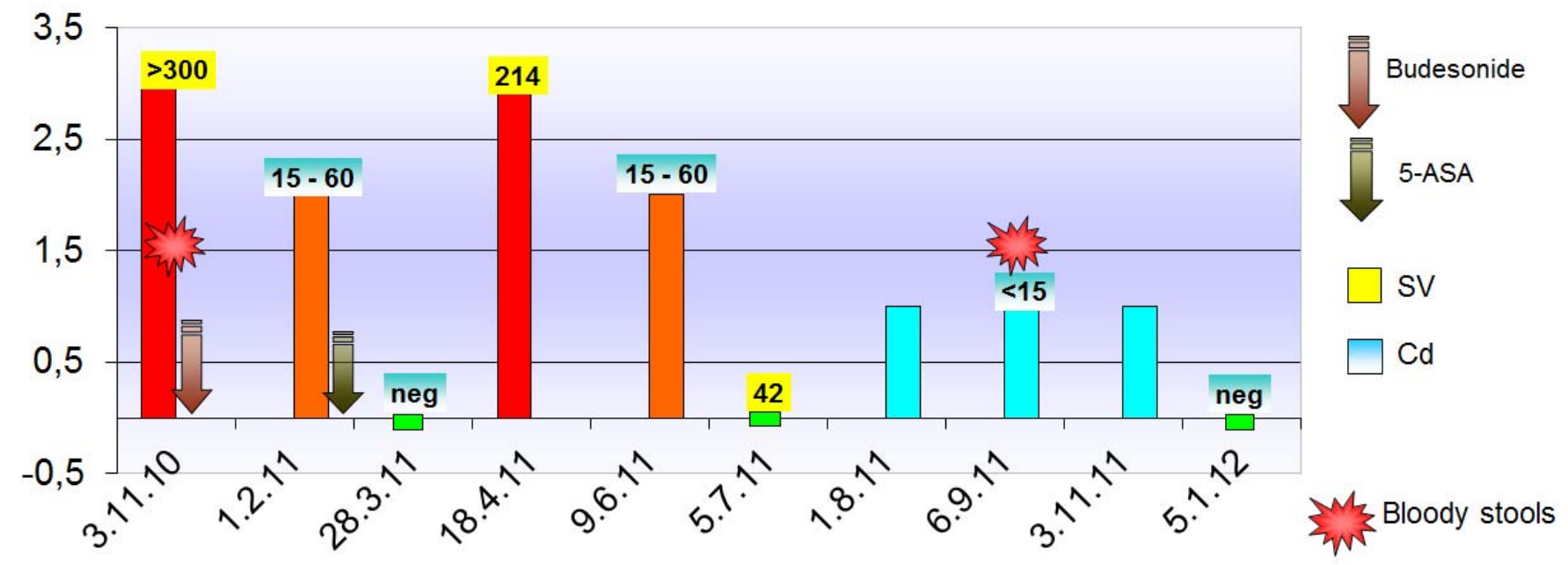

Fig. 5. Calprotectin

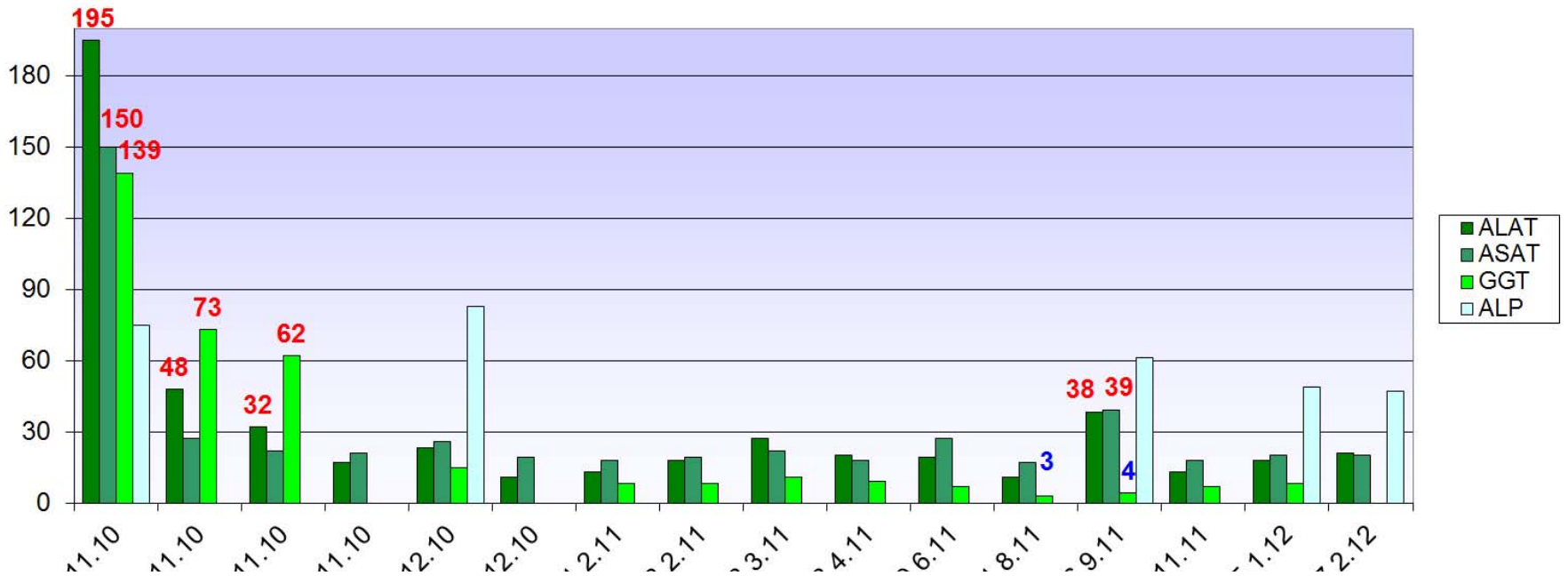

Fig. 6. Hepatic tests

munoregulation in intestinal mucosal may be of primary importance in the pathogenesis. The gut is under constant immunologic stimulation and displays "physiologic" inflammation but when this fails to be controlled, pathologic inflammation ensues.

PSC is a chronic autoimmune cholestatic liver disease similar to PBC, except that in PSC the bile ducts both within and outside the liver become narrowed, due to inflammation and scarring. The incidence of PSC in children has dramatically increased [8]. According to Woodward and Neuberger [3] it is highly associated with IBD, is characterised by ANCA antibodies, affects the small and the large bile ducts as well, and does not respond to corticosteroids. It is diagnosed in patients with signs of cholestatic 


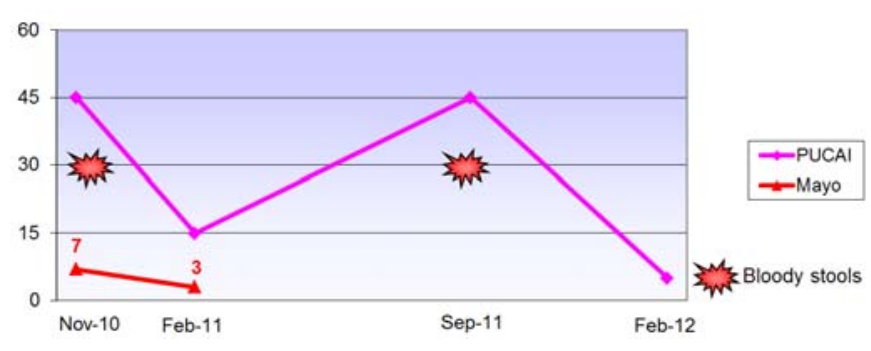

Fig. 7. PUCAI and Mayo endoscopic score

liver disease, (elevated ALP and GGT levels) and underlying bile duct abnormalities (seen on liver biopsy, sensitive imaging tests like cholangiography, magnetic resonance cholangiography (MRC), ultrasonography, or MRI, which may be accompanied by endoscopy [8]. PBC is unique among autoimmune disease in that the condition has not been described in children [3]. AIH is due to a faulty immune system that attacks the body's own cells [9]. Based on the scoring system developed by International Autoimmune Hepatitis Group [IAIHG] [10,11] the key diagnostic components are elevated serum transaminases, biopsy evidence of interface hepatitis with or without lobular hepatitis or central-portal bridging necrosis and the presence of specific autoantibodies (ANA, SMA, LKM, SLA). Several conditions like drug-induced hepatitis or viral infection need to be effectively excluded before a definite diagnosis can be made. Czaja AJ reported that overlapping autoimmune diseases of the liver are present in about $18 \%$ and about $5 \%$ of patients with a primary diagnosis of AIH have signs and symptoms of PBC (bilirubin and ALP elevation, liver biopsy) [12]. The syndrome of autoimmune cholangiopathy is still subject for discussion. It can be described as a subentity of AIH type1 [13] or as an AMA-negative form of PBC [14]. SMA are directed against cytoskeletal proteins such as actin, troponin, and tropomyosin $[15,16]$. They are not highly specific for AIH and have been shown to occur in advanced liver diseases of other etiologies, in infectious diseases and rheumatic disorders but in pediatric patients, SMA autoantibodies may be the only marker of AIH type 1 [17].

In order to confirm diagnosis,we performed serologic tests. We found only ANCA and SMA positive. In a study that evaluated the sensitivity and specificity of four antibodies in children with IBD they found ANCA positive in $70 \%$ of children with UC and only in $18 \%$ of those with CD. ASCA instead was highly specific for CD and children were more likely to have disease of the ileum or ileum and right colon than patients who were ASCA-negative (58\% vs $18 \%, \mathrm{p}<0.001)$ [18]. Our patient was ASCA negative but had involvement of ileum and right colon. This may be explained by the fact that the author concludes that ASCA identifies predominantly the subset of children with disease of the ileum and ascending colon who may be at increased risk of surgery.

Anemia is a common finding in chronic inflammations and it may be explained by the variety of inflammatory cytokines that can interfere with erythropoiesis and by iron deficiency that is secondary to chronic blood loss as well as a decreased intake [1]. We found high ESR but a low CRP. This in concordance with reported data that characterizes CRP as less reliable marker of inflammation and disease activity in patients with UC, and this fact is in contrast with other gastrointestinal inflammations such as $\mathrm{CD}$ and acute pancreatitis, where its levels correlate well with clinical disease activity [19]. We already reported two cases of Crohn disease with high CRP levels that correlated with clinical disease activity $[20,21]$. Fecal calprotectin was reported to correlate well with disease activity. It was first isolated by Fagerhol and is an important component of neutrophil cytosol. He first named it L1 protein, later was renamed due to its property of binding calcium. This way protects the cell from catalytic enzymes [22].Calprotectin pass into the intestinal lumen when leucocytes are activated or destroyed [23]. It has been proposed as a useful tool in differentiating organic diseases of colon from IBS [24]. We had high levels at the beginning, but in September didn't rise as much as ESR did, although blood reappeared in stools and PUCAI had the same value as in November 2010.

It is strongly recommended to rule out any infectious colitis before establishing the diagnosis and to consider the possibility of enteric infection with recurrent symptoms cause these infections may mimic a flare-up or actually provoke a recurrence of UC [1]. We performed repeated bacteriological tests to search such an infection, but all coprocytogram and stool cultures were negative.

Budesonide, as described by the producer, is a synthetic corticosteroid, has a high topical glucocorticosteroid (GCS) activity and substantial first pass elimination. The formulation contains granules which are coated to protect dissolution in gastric juice, but which dissolve when the granules reach the duodenum. Systemic availability after a single dose is higher in patients with CD compared to healthy volunteers, $(21 \%$ vs. $9 \%)$ but approaches that in healthy volunteers after repeated dosing. After absorption, budesonide is subject to high first pass metabolism(80-90\%).In patients with liver cirrhosis, systemic availability of orally administered Budesonide correlates with disease severity and is, on average, 2.5-fold higher compared with healthy controls. Patients with mild liver disease are minimally affected.

The Gastrointestinal Drugs Advisory Committee to the US Food and Drug Administration (FDA) recommended in July 2011 Infliximab to be approved for the treatment of moderate to severe active ulcerative colitis in children. But there are still concerns about this therapy especially when used long term like immunogenicity and malignancy. Rachel Rosen, MD, from Children's Hospital Boston in Massachusetts highlighted that more safety data are definitely needed. Currently in Romania Infliximab is not approved for treating UC in children.

We were successful in treatment with Budesonide and 5-ASA, but we have to be aware that UC is a chronic dis- 
ease and beyond the $1^{\text {st }}$ decade of disease, the risk to develop colon cancer increase rapidly. After treatment of initial symptoms, $\approx 5 \%$ of children with UC have a prolonged remission ( $>3 \mathrm{yr}$ ). About $25 \%$ of children presenting with severe ulcerative colitis require colectomy within $5 \mathrm{yr}$ of diagnosis, compared with only $5 \%$ of those presenting with mild disease. The risk of colon cancer may be diminished with surveillance colonoscopies beginning after $8-10 \mathrm{yr}$ of disease [1].

\section{Conclusions}

Ulcerative colitis may have variable onsets, we had in this case a simultaneous onset of diarrhea with blood in stools and extraintestinal manifestations, or we could consider the previous manifestation of irritable bowel syndrome like the real onset of UC.

The immunological markers didn't fully fit any of the associated diseases profiles, so we concluded that there was an overlap syndrome.

We had a high AIH diagnostic score, although we couldn't make a complete count due to lack of hepatic biopsy data.

Regarding our patient Budesonide was effective on both hepatic and intestinal disease. Cholestatic syndrome, although incompletely confirmed by blood tests (normal ALP levels) was rapidly improved with treatment.

\section{References}

1. Hyams JS. Inflammatory Bowel Disease. in: Kliegman RM, Behrman RE, Jenson HB, Stanton BF. ed. Nelson Text Took of Pediatrics. 18 th ed. New Delhi: Elsevier Inc.; 2007:1575-85.

2. Xanthakos SA, Balistreri WF. Liver Disease Associated with Systemic Disorders. in: Kliegman RM, Behrman RE, Jenson HB, Stanton BF. ed. Nelson Text Took of Pediatrics. 18 th ed. New Delhi: Elsevier Inc.; 2007:1692-5.

3. Woodward J, Neuberger J. Autoimmune overlap syndromes. Hepatology. 2001 Apr;33(4):994-1002.

4. Hubscher G. Pathology of vanishing bile duct syndromes. in: Neuberger $\mathrm{J}$ ed. Primary Biliary Cirrhosis. Eastbourne: West End Studios; 2000. p. 41-52.

5. Mackay I-R. Historical reflections on autoimmune hepatitis. World J. Gastroenterol. 2008 Jun 7;14(21):3292-300.

6. Turner D, Otley AR, Mack D, Hyams J, de Bruijne J, Uusoue K, et al. Development, Validation, and Evaluation of a Pediatric Ulcerative Colitis Activity Index: A Prospective Multicenter Study. Gastroenterology. 2007 Aug;133(2):423-32.
7. Schroeder KW, Tremaine WJ, Ilstrup DM. Coated oral 5-aminosalicylic acid therapy for mildly to moderately active ulcerative colitis. A randomized study. N. Engl. J. Med. 1987 Dec 24;317(26):1625-9.

8. Primary Sclerosing Cholangitis. suite101.com [Internet]. [cited 2012 Apr 4]; Available from: http://elaine-moore.suite101.com/primary-sclerosingcholangitis-a13444

9. Autoimmune Hepatitis. suite101.com [Internet]. [cited 2012 Apr 7]; Available from: http://wendyamprosser.suite101.com/autoimmunehepatitis-a202937

10. Alvarez F, Berg PA, Bianchi FB, Bianchi L, Burroughs AK, Cancado EL, et al. International Autoimmune Hepatitis Group Report: review of criteria for diagnosis of autoimmune hepatitis. J. Hepatol. 1999 Nov;31(5):929-38.

11. Johnson PJ, McFarlane IG. Meeting report: International Autoimmune Hepatitis Group. Hepatology. 1993 Oct;18(4):998-1005.

12. Czaja AJ. Frequency and nature of the variant syndromes of autoimmune liver disease. Hepatology. 1998 Aug;28(2):360-5.

13. Ben-Ari Z, Dhillon AP, Sherlock S. Autoimmune cholangiopathy: part of the spectrum of autoimmune chronic active hepatitis. Hepatology. 1993 Jul;18(1):10-5.

14. Colombato LA, Alvarez F, Côté J, Huet PM. Autoimmune cholangiopathy: the result of consecutive primary biliary cirrhosis and autoimmune hepatitis? Gastroenterology. 1994 Dec;107(6):1839-43.

15. Czaja AJ, Cassani F, Cataleta M, Valentini P, Bianchi FB. Frequency and significance of antibodies to actin in type 1 autoimmune hepatitis. Hepatology. 1996 Nov;24(5):1068-73.

16. Toh $\mathrm{BH}$. Smooth muscle autoantibodies and autoantigens. Clin Exp Immunol. 1979 Dec;38(3):621-8.

17. Strassburg CP, Manns MP. Autoantibodies and Autoantigens in Autoimmune Hepatitis. Seminars in Liver Disease. 2002;22(4):339-52.

18. Zholudev A, Zurakowski D, Young W, Leichtner A, Bousvaros A. Serologic testing with ANCA, ASCA, and anti-OmpC in children and young adults with Crohn's disease and ulcerative colitis: diagnostic value and correlation with disease phenotype. Am. J. Gastroenterol. 2004 Nov;99(11):2235-41.

19. Vermeire S, Assche GV, Rutgeerts P. The role of C-reactive protein as an inflammatory marker in gastrointestinal diseases. Nature Reviews Gastroenterology and Hepatology. 2005 Dec 1;2(12):580-6.

20. A H, I S, I S, Oana B, Andrea M. Successful Treatment with Infliximab in a Case of Crohn's Disease with Peripheral Arthropathyes. Acta Medica Marisiensis. 2011 Apr 1;57(3):256-9.

21. Hodut A, Simedrea I, Belei O, Militaru A, Raluca B, Brad G. Acute Infusion Reaction to Infliximab in a Case of Crohn's Disease with Recto-Scrotal Fistula. Acta Medica Marisiensis. 2011 Dec 7;57(5):544-7.

22. Fagerhol MK, Dale I, Anderson T. Release and Quantitation of a Leucocyte Derived Protein (L1). Scandinavian Journal of Haematology. 1980 Dec 1;24(5):393-8.

23. Steinbakk M, Naess-Andresen CF, Lingaas E, Dale I, Brandtzaeg P. Fagerhol MK. Antimicrobial actions of calcium binding leucocyte L1 protein, calprotectin. Lancet. 1990 Sep 29;336(8718):763-5.

24. El Saadany Sherif, Wael Farrag Mohamed, Abdullah A. Mohamed, Sahar A.F. Hammoudah. Fecal Calprotectin as a Marker in Differentiating Irritable Bowel Syndrome from Organic Intestinal Disease. elsadany66 [Internet]. [cited 2012 Apr 3]; Available from: http://elsadany66.wordpress.com/ article/fecal-calprotectin-as-a-marker-in-i2p6c6e8rrui-7 